\title{
Resonant Tunnelling through InAs Quantum Dots in Tilted Magnetic Fields: Experimental Determination of the g-factor Anisotropy
}

\author{
J. M. Meyer (a), I. Hapke-Wurst (a), \\ U. Zeitler (a), R. J. Haug (a), and K. Pierz (b) \\ (a) Institut für Festkörperphysik, Universität Hannover, \\ Appelstraße 2, 30167 Hannover, Germany \\ (b) Physikalisch-Technische Bundesanstalt Braunschweig, \\ Bundesallee 100, 38116 Braunschweig, Germany
}

(Submitted July 14, 2000)

subject classification: $73.40 \mathrm{Gk}, 73.23 \mathrm{Hk}, 73.61 \mathrm{Ey}$; S7.12

\begin{abstract}
We have determined the Landé factor $g^{*}$ in self-organized InAs quantum dots using resonant-tunnelling experiments. With the magnetic field applied parallel to the growth direction $z$ we find $g_{\|}^{*}=0.75$ for the specific dot investigated. When the magnetic field is tilted away by $\vartheta$ from the growth axis, $g^{*}$ gradually increases up to a value $g_{\perp}^{*}=0.92$ when $B \perp z$. Its angular dependence is found to follow the phenomenological behaviour $g^{*}(\vartheta)=\sqrt{\left(g_{\|}^{*} \cos \vartheta\right)^{2}+\left(g_{\perp}^{*} \sin \vartheta\right)^{2}}$.
\end{abstract}

Introduction Resonant tunnelling experiments through zero-dimensional structures are an efficient tool to access their quantized energy levels. In recent years several groups succeeded in performing such experiments with self-assembled InAs quantum dots (QDs) embedded in the barrier of a single-barrier tunnelling device [1, 2]. Low-temperature experiments allowed to measure directly the Landé factor $g^{*}$ of InAs dots [3, in. In this Note we will report on resonant tunnelling experiments through InAs QDs in tilted magnetic fields. We will show that $g^{*}$ depends on the orientation of the magnetic field with respect to the growth direction and can be described phenomenologically by a tensor with two independent components.

Sample structure The samples were grown by molecular-beam epitaxy on a highly Sidoped GaAs substrate with a donor concentration $n=2 \times 10^{18} \mathrm{~cm}^{-3}$. First we grew a 1- $\mu \mathrm{m}$ thick GaAs buffer layer with the same doping level followed by two 10-nm thick ndoped GaAs layers with $n=1 \times 10^{17} \mathrm{~cm}^{-3}$ and $n=1 \times 10^{16} \mathrm{~cm}^{-3}$ and an 15-nm thick undoped GaAs spacer. On this bottom electrode a 10-nm thick AlAs barrier was deposed. The growth of the barrier was interrupted at a thickness of $5 \mathrm{~nm}$ where 1.8 mono-layers of InAs were embedded in the barrier. With such an InAs coverage self-assembled InAs QDs are formed. The structure was terminated with a top electrode symmetric to the bottom electrode finishing with $1 \mu \mathrm{m}$ highly n-doped GaAs $\left(n=2 \times 10^{18} \mathrm{~cm}^{-3}\right)$. As a consequence of the high doping three-dimensional electrodes are present on both sides of the AlAs barrier.

Subsequent to the growth of the wafer macroscopic AuGeNi contacts with a typical diameter of $50 \mu \mathrm{m}$ were annealed into the top electrode and vertical tunnelling diodes with the same diameter were processed using wet-chemical etching.

Resonant Tunnelling and Spin Splitting When a bias voltage is applied between the top and the bottom electrode our devices show $I-V$-characteristics which can be described by tunnelling through a single AlAs barrier [2]. Superimposed on this coarse $I$ - $V$-curve 


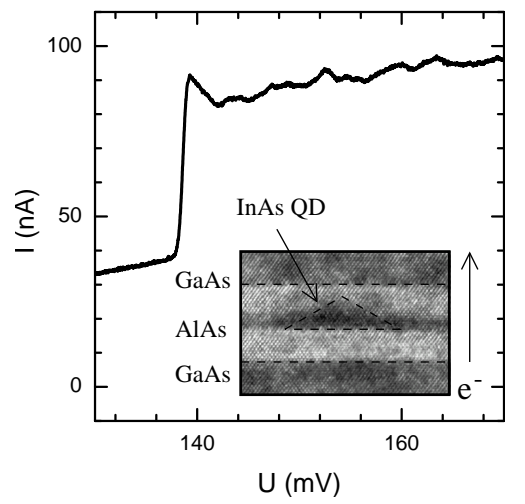

Figure 1: ( Small secCion of the $I-V$-curve of a single barrier GaAs-AlAs-GaAs tunnelling device with InAs dots embedded in the middle of the AlAs barrier. The structure of a reference sample is shown in the transmission electron micrograph in the inset. At positive bias voltages the electrons tunnel from the bottom to the top as indicated by the arrow. The step observed is due to single electron tunnelling through an individual InAs quantum dot.

we observe distinct current steps assigned to resonant tunnelling through individual InAs quantum dots [⿰], for more details see 2, 4]. A typical step for $T=0.5 \mathrm{~K}$ is shown in Fig. 1 .

In high magnetic fields the current step observed at $B=0 \mathrm{~T}$ develops into two spin-split steps with a voltage separation $\Delta V=g^{*} \mu_{B} B / \alpha e$. Here $\alpha=E_{D} / e V$ denotes the lever factor between the energy separation emitter-dot, $E_{D}$, and the total voltage drop, $V$. From the temperature dependent smearing of the current step we deduced $\alpha=0.3$. The spin splitting of the current step is shown in Fig. 2 a where the $I$ - $V$-characteristic of the tunnelling structure at $B=10 \mathrm{~T}$ is displayed. For the top curve the magnetic field $B$ is oriented along the growth direction $z$, the bottom trace was measured in a magnetic field perpendicular to $z$.

As can be seen in Fig. 2b, $\Delta V$ indeed increases linearly with magnetic field confirming the scenario of a simple Zeeman splitting of the QD's energy level. However, the Landé factor $g^{*}$ as deduced from the slope is considerably different for the two field orientations $B \| z$ and $B \perp z$ where we find Landé factors $g_{\|}^{*}=0.74$ and $g_{\perp}^{*}=0.92$. From the temperature dependence of the step heights in high magnetic fields we find that the low-voltage step can be assigned to tunnelling of spin-down electrons 4 - resulting in a positive $g$-factor as also found in 3].

At first sight it is quite astonishing that the Landé factor is far away from that of bulk InAs $\left(g^{*}=-14.8\right)$. This discrepancy can be explained qualitatively considering effects of size quantization, strain and possible other effects [3]. Such effects may be the leakage of the electronic wave-function in the InAs quantum dot into the AlAs barrier and the space dependent alloying of the InAs dots with AlAs. In a simple picture this can be described by an (rather complex) admixture of the Landé factor in AlAs and strained InAlAs to the (size-quantized) $g$-factor in the InAs-dot.

Tilted Magnetic Fields In order to clarify the dependence of $g^{*}$ on the orientation of the magnetic field more clearly we have performed experiments in tilted magnetic fields at a

\footnotetext{
${ }^{\dagger}$ We obtained similar results for a second step observed at higher bias voltages. This step can be assigned to the tunneling through another InAs QD. For the two field orientations we find $g_{\|}^{*}=0.78$ and $g_{\perp}^{*}=0.99$.
} 

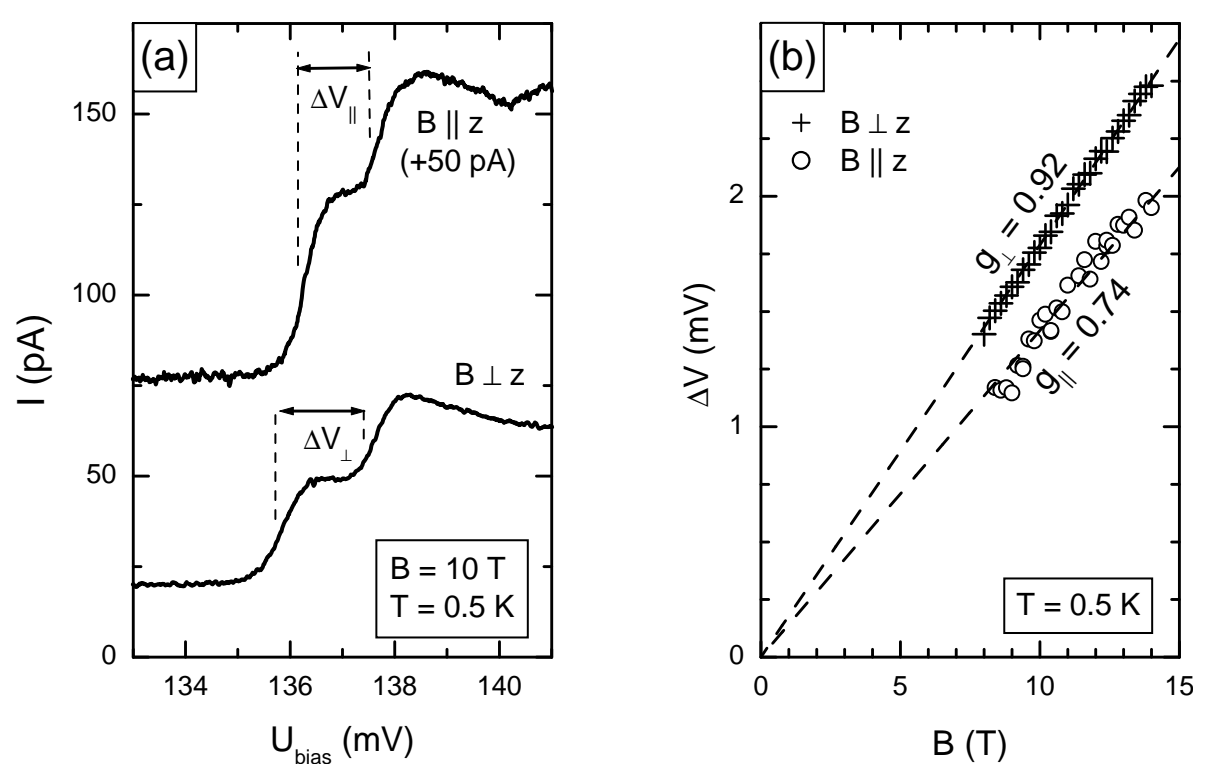

Figure 2: (a) Spin splitting of the current step shown in Fig. 1 for $B=10 \mathrm{~T}$ at $T=0.5 \mathrm{~K}$ for two field orientations. The top trace is shifted for clarity.

(b) Dependence of the spin splitting $\Delta V$ on the magnetic field for both field orientations.

temperature $T=1.3 \mathrm{~K}$. The angle between the magnetic field and the growth direction was stepped from $\vartheta=0^{\circ}$ to $\vartheta=90^{\circ}$. For each angle I-V-curves were recorded at $B=15 \mathrm{~T}$ and the voltage splitting between two spin-split steps was determined. From this we deduced the angular dependent Landé factor, $g^{*}(\vartheta)$. The results are shown in Fig. 3. As can be seen in the figure, $g^{*}(\vartheta)$ gradually increases when tilting the magnetic field away from the growth axis. As indicated with the solid line it follows an angular dependence

$$
g^{*}(\vartheta)=\sqrt{\left(g_{\|}^{*} \cos \vartheta\right)^{2}+\left(g_{\perp}^{*} \sin \vartheta\right)^{2}},
$$

with $g_{\|}^{*}=0.74$ and $g_{\perp}^{*}=0.92$.

This phenomenological behaviour can be understood when regarding the Zeeman contribution to the total energy in a size quantized structure. For the most general case the corresponding Hamiltonian can be written in the form [5]

$$
\mathcal{H}_{Z}=\frac{1}{2} \mu_{B} \sigma_{\alpha} g_{\alpha \beta} B_{\beta},
$$

were $\alpha=x, y, z$ are the three spatial directions, $B_{\alpha}$ are the components of the magnetic field along $\alpha, \mu_{B}$ is the Bohr magneton and $\sigma_{\alpha}$ are the Pauli spin-matrices. In the most general form the tensor $g_{\alpha \beta}$ contains nine independent real components [5].

To get a better physical insight we model our dot by a flat disc with a height $h \approx 3 \mathrm{~nm}$ and a diameter $d \approx 15 \mathrm{~nm}[2]$. In this case the Landé tensor reduces to a diagonal tensor with two independent components $g_{\perp}^{*}=g_{x x}=g_{y y}$ and $g_{\|}^{*}=g_{z z}$. In a tilted magnetic field with $B_{x}=0, B_{y}=B \sin \vartheta$ and $B_{z}=B \cos \vartheta$ equation (14) then directly follows from (2).

Discussion Our experiment clearly shows the anisotropic nature of spin splitting in InAs quantum dots. Comparing with the measured $g$-factor anisotropy in quantum-wires [6] and 


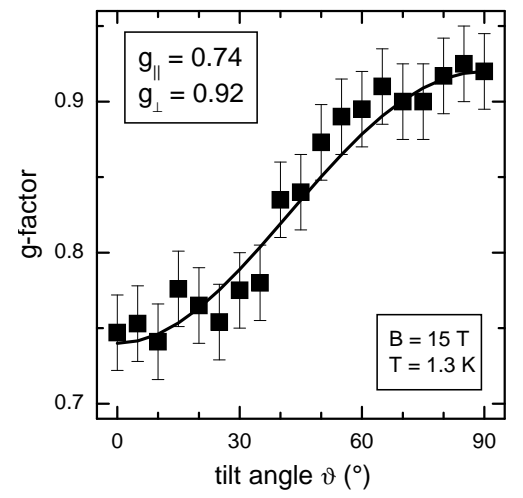

Figure 3: Angular dependence of the experimentally measured $g^{*}$. The dots show the experimental values, the solid line is a phenomenological fit with two independent tensor components of the $g$-factor tensor.

quantum wells [7] one would expect the largest $g$-factor when the magnetic field is applied in the direction of the strongest confinement. Our experiments, however, indicate that this simple relation for the $g$-factor anisotropy does not hold. We observe a smaller $g^{*}$ if the magnetic field is applied in the growth direction where the quantum confinement is the strongest. Therefore, it is probable that the effects responsible for the $g$-factor anisotropies are not dominated by quantum confinement but by more complicated mechanisms such as AlAs alloying into the InAs quantum dots, leakage of the electronic wave function into the AlAs barriers, spatial dependent strain, spin interactions between the dot and the electrodes etc. Without any doubt more elaborate theoretical models are necessary to clarify the possible origins of $g$-factor anisotropies in self-assembled InAs quantum dots.

Conclusions Using resonant tunnelling experiments we have measured the Landé factor tensor of InAs quantum dots. The $g$-factor tensor was described by two independent tensor components $g_{\|}^{*}$ and $g_{\perp}^{*}$.

\section{References}

[1] I. E. Itskevich, T. Ihn, A. Thornton, M. Henini, T. J. Foster, P. Moriarty, A. Nogaret, P. H. Beton, L. Eaves, and P. C. Main, Phys. Rev. B 54, 16401 (1996);

T. Suzuki, K. Nomoto, K. Taira, and I. Hase, Jpn. J. Appl. Phys. 36, 1917 (1997);

M. Narihiro, G. Yusa, Y. Nakamura, T. Noda, and H. Sakaki, Appl. Phys. Lett. 70, 105 (1997).

[2] I. Hapke-Wurst, U. Zeitler, H. W. Schumacher, R. J. Haug, K. Pierz, and F. J. Ahlers, Semicond. Sci. Technol. 14, L41 (1999).

[3] A. S. G. Thornton, T. Ihn, P. C. Main, L. Eaves, and M. Henini, Appl. Phys. Lett. 73, 354 (1998).

[4] I. Hapke-Wurst, U. Zeitler, H. Frahm, A. G. M. Jansen, R. J.Haug, and K. Pierz, accepted for publication in Phys. Rev. B (see also cond-mat/0003400)

[5] A. A. Kiselev, E. L. Ivchenko, and U. Rössler, Phys. Rev. B 58, 16353 (1998).

[6] M. Oestreich, A. P. Heberle, W.W. Rühle, R. Nötzel, and K. Ploog, Europhys. Lett. 31, 399 (1995).

[7] P. Le Jeune, D. Robart, X. Marie, T. Amand, M. Brousseau, J. Barrau, V. Kalevich, and D. Rodichev, Semicond. Sci. Technol. 12, 380 (1997). 